\title{
ANOTHER LOOK AT THE LANDAU THEOREM
}

\author{
Zhenghua Xu \\ Hefei University of Technology, School of Mathematics \\ Hefei 230601, P. R. China; zhxu@hfut.edu.cn
}

\begin{abstract}
Let $f$ be the analytic self-map of the open unit disk $\mathbf{D}$ in complex plane with $f(0)=0$ and $f^{\prime}(0) \neq 0$. The classical Landau theorem shows that the image $f(\mathbf{D})$ contains a schlicht disk. In this note, it is proved that the injectivity of $f$ in the Landau theorem can be strengthened to be starlike. In particular, it provides a way to construct starlike functions from bounded analytic functions. Furthermore, we obtain a new version of the Landau theorem for vector-valued analytic functions from the perspective of modulus functions.
\end{abstract}

\section{Introduction}

Denote by $\mathbf{D}$ the open unit disk of the complex plane $\mathbf{C}$. If $f: \mathbf{D} \rightarrow \mathbf{D}$ is an analytic function normalized by $f(0)=0$ and $\left|f^{\prime}(0)\right|=\alpha>0$, then the classical Landau theorem states that $f$ is injective on $r_{0} \mathbf{D}$ with $r_{0}=\alpha /\left(1+\sqrt{1-\alpha^{2}}\right)$ and $f\left(r_{0} \mathbf{D}\right)$ contains the disk $R_{0} \mathbf{D}$ with $R_{0}=r_{0}^{2}$, [13]. Furthermore, $r_{0}$ and $R_{0}$ are both sharp with the extremal function $f(z)=z(z+\alpha) /(1+z \alpha)$. Historically, the Landau theorem can be used to prove the celebrated Bloch theorem saying that $f(\mathbf{D})$ contains a schlicht disk of universal radius for any analytic function $f$ with the only normalization $f^{\prime}(0)=1$, see, e.g., [8].

Recently, the Landau theorem has been studied extensively; see e.g. [16] for mappings of bounded and finite distortion, $[5,6,7,9,11,14]$ for harmonic mappings and $[1,3,4,10]$ for holomorphic or pluriharmonic mappings. However, all of these extensions and generalizations are not sharp. The sharp version of the Landau theorem has been generalized into slice regular functions over quaternions in [2] and [21], independently.

Let $\Omega$ be a starlike domain with respect to the origin, that is $t \Omega \subset \Omega$ for all $t \in[0,1]$. For the analytic function $f$ on $\Omega$ with $f(0)=0, f$ is said to be starlike on $\Omega$ if $f$ is injective on $\Omega$ and the image $f(\Omega)$ is starlike with respect to the origin. In fact, the injectivity of $f$ in the Landau theorem can be strengthened to be starlike on $r_{0} \mathbf{D}$ as shown by Theorem 1.1 and then $f(\mathbf{D})$ contains a schlicht disk by the $\frac{1}{4}$-covering theorem.

For the complex-valued analytic function $f$ on $\mathbf{D}$ with $f(0)=0$ and $f^{\prime}(0) \neq 0$, it is well known that the analytical characterization of starlikeness on $\mathbf{D}$ can be presented as

$$
\operatorname{Re} \frac{z f^{\prime}(z)}{f(z)}>0, \quad \forall z \in \mathbf{D}
$$

https://doi.org/10.5186/aasfm.2021.4644

2020 Mathematics Subject Classification: Primary 46E40; Secondary 30C25, 30C45.

Key words: Vector-valued analytic function, Landau theorem, starlike function.

This work was supported by the National Natural Science Foundation of China (No. 11801125) and the Fundamental Research Funds for the Central Universities (Nos. JZ2019HGTB0054 and JZ2018HGBZ0118). 
In fact, the formula above is also equivalent to that the modulus function $M(r)=$ $|f(r u)|$ is strictly increasing on $[0,1)$ for each $u \in \partial \mathbf{D}$. Furthermore, this conclusion holds also for slice starlike functions over quaternions [20]. From this perspective, we shall in this note establish a new and sharp version of the Landau theorem for vector-valued analytic functions as follows.

Theorem 1.1. Let $f: \mathbf{D} \rightarrow \mathbf{C}^{n}$ be a vector-valued analytic function with $f(0)=$ $0,\left|f^{\prime}(0)\right|=\alpha>0$ and $|f(z)|<1$ for all $z \in \mathbf{D}$. Then, for any $\theta \in \mathbf{R},\left|f\left(r e^{i \theta}\right)\right|$ and $r^{2} /\left|f\left(r e^{i \theta}\right)\right|$ are strictly increasing for $r \in\left(0, r_{0}\right)$, where $r_{0}=\alpha /\left(1+\sqrt{1-\alpha^{2}}\right)$ is sharp.

Theorem 1.1 provides a method of constructing starlike functions from bounded analytic functions.

Corollary 1.2. If $f$ is a complex-valued analytic function on $\mathbf{D}$ with $f(0)=$ $0,\left|f^{\prime}(0)\right|=\alpha>0$ and $|f(z)|<M$ for all $z \in \mathbf{D}$, then $f(z)$ and $z^{2} / f(z)$ are starlike functions on $r \mathbf{D}$ with $r=\alpha /\left(M+\sqrt{M^{2}-\alpha^{2}}\right)$.

Remark 1.3. In the proof of Theorem 1.1, we show directly the starlikeness of $f$ on $r_{0} \mathbf{D}$ which means naturally that $f$ is injective on $r_{0} \mathbf{D}$ for complex-valued analytic functions. This approach is totally different from the usual case; see for instance [3, Theorem 1].

Remark 1.4. In the case of several complex variables, it is worth mentioning that the starlikeness of analytic functions is not equivalent to that its modulus is increasing in the radial direction. To see this, we take the vector-valued analytic function $f(z, w)=(z+a z w, w)$ on the open unit ball $B^{2}=\left\{(z, w) \in \mathbf{C}^{2}:|z|^{2}+|w|^{2}<\right.$ $1\}$. For any $a \in \overline{\mathbf{D}}, f$ is injective on $B^{2}$ and $f\left(B^{2}\right)$ is a starlike domain with respect to the origin [17, Example 6]. Hence, $f$ is starlike on $B^{2}$. However, $|f(z, w)|$ is not increasing in the radial direction. In fact, $|f(z, w)|$ is given by

$$
|f(z, w)|^{2}=\left|z^{\prime} w^{\prime} a\right|^{2} r^{4}+2 \operatorname{Re}\left(a w^{\prime}\right)\left|z^{\prime}\right|^{2} r^{3}+r^{2},
$$

with $(z, w)=r\left(z^{\prime}, w^{\prime}\right), r=\sqrt{|z|^{2}+|w|^{2}} \geq 0$, and $\left(z^{\prime}, w^{\prime}\right) \in \partial B^{2}$.

Then, we obtain

$$
\frac{\partial}{\partial r}|f(z, w)|^{2}=2 r\left(2\left|z^{\prime} w^{\prime} a\right|^{2} r^{2}+3 \operatorname{Re}\left(a w^{\prime}\right)\left|z^{\prime}\right|^{2} r+1\right)
$$

is negative for some $r \in(0,1)$ since the discriminant $\triangle=\left|z^{\prime}\right|^{2}\left(9\left|z^{\prime}\right|^{2}\left(\operatorname{Re}\left(a w^{\prime}\right)\right)^{2}-\right.$ $\left.8\left|a w^{\prime}\right|^{2}\right)$ takes some positive values.

The next section shall be devoted to the proof of our main result (Theorem 1.1).

\section{Proof of Theorem 1.1}

In order to prove Theorem 1.1, we need two lemmas.

Lemma 2.1. If $f: \mathbf{D} \rightarrow \mathbf{C}^{n}$ is a vector-valued analytic function satisfying $|f(z)|<1$ for all $z \in \mathbf{D}$, then

$$
\left|\left\langle f^{\prime}(z), f(z)\right\rangle\right| \leq|f(z)| \frac{1-|f(z)|^{2}}{1-|z|^{2}} .
$$

In fact, for $f(z) \neq 0,(2.1)$ can be rewritten as

$$
|\nabla| f(z)|| \leq \frac{1-|f(z)|^{2}}{1-|z|^{2}}
$$


which was first proved by Pavlović [15] and generalised by the author for analytic functions of the open unit ball of JB*-triples into Banach spaces [19]. The interested readers may refer to $[12,18]$ for the harmonic and pluriharmonic versions of Lemma 2.1, respectively.

Lemma 2.2. If $f: \mathbf{D} \rightarrow \mathbf{C}^{n}$ is a vector-valued analytic function satisfying $|f(z)|<1$ for all $z \in \mathbf{D}$, then

$$
\frac{|f(0)|-|z|}{1-|z||f(0)|} \leq|f(z)| \leq \frac{|z|+|f(0)|}{1+|z||f(0)|}, \quad \forall z \in \mathbf{D} .
$$

Proof. Fix $l \in \mathbf{C}^{n}$ with $|l|=1$ and consider the analytic self-map of $\mathbf{D}$ given by $g(z)=\langle f(z), l\rangle$. Applying the classical Schwarz-Pick lemma to the complex-valued analytic function $g$, we have, for all $z \in \mathbf{D}$,

$$
\left|\frac{g(z)-g(0)}{1-g(z) \overline{g(0)}}\right| \leq|z|
$$

Equivalently,

$$
\left|g(z)-\frac{\left(1-|z|^{2}\right) g(0)}{1-|z|^{2}|g(0)|^{2}}\right| \leq \frac{|z|\left(1-|g(0)|^{2}\right)}{1-|z|^{2}|g(0)|^{2}}
$$

which implies by the triangle inequality that

$$
\frac{|g(0)|-|z|}{1-|z||g(0)|} \leq|g(z)| \leq \frac{|z|+|g(0)|}{1+|z||g(0)|}
$$

Note that, for $a \in(0,1)$, the function $h(x)=\frac{a+x}{1+a x}$ is increasing for $x \in(0,1)$. Hence the inequality $|g(0)|=|\langle f(0), l\rangle| \leq|f(0)|$ shows that

$$
|g(z)| \leq \frac{|z|+|g(0)|}{1+|z||g(0)|} \leq \frac{|z|+|f(0)|}{1+|z||f(0)|}
$$

The right inequality in (2.2) is trivial if $f(z)=0$ and otherwise obtained by taking $l=f(z) /|f(z)|$ in (2.4). On the other hand, (2.3) implies also that

$$
\frac{|g(0)|-|z|}{1-|z||g(0)|} \leq|g(z)| \leq|f(z)| \text {. }
$$

The left inequality in (2.2) is trivial if $f(z)=0$ and otherwise can be obtained by taking $l=f(0) /|f(0)|$ in $(2.5)$.

We are now in a position to prove the main result.

Proof of Theorem 1.1. Consider the analytic function $h: \mathbf{D} \rightarrow \mathbf{C}^{n}$ given by

$$
h(z)= \begin{cases}z^{-1} f(z), & \text { if } z \in \mathbf{D} \backslash\{0\} \\ f^{\prime}(0), & \text { if } z=0\end{cases}
$$

By applying the maximum modulus principle for vector-valued analytic functions, we have $|h(z)| \leq 1$ for all $z \in \mathbf{D}$. Lemma 2.1 states that

$$
\left|\left\langle h^{\prime}(z), h(z)\right\rangle\right| \leq|h(z)| \frac{1-|h(z)|^{2}}{1-|z|^{2}}, \quad \forall z \in \mathbf{D} .
$$

From the left inequality in (2.2), for $|z|=r<\alpha$, we have $h(z) \neq 0$ and

$$
\left|\left\langle h^{\prime}(z), \frac{h(z)}{|h(z)|}\right\rangle\right| \leq \frac{1-|h(z)|^{2}}{1-r^{2}} \leq \frac{1}{1-r^{2}}\left(1-\left(\frac{\alpha-r}{1-r \alpha}\right)^{2}\right)=\frac{1-\alpha^{2}}{(1-\alpha r)^{2}} .
$$


Note that, for $r \in\left(0, r_{0}\right)$, it holds that $\alpha r^{2}-2 r+\alpha>0$. Equivalently,

$$
\frac{r\left(1-\alpha^{2}\right)}{(1-\alpha r)^{2}}<\frac{\alpha-r}{1-\alpha r}, \quad r \in\left(0, r_{0}\right) \text {. }
$$

Hence, Lemma 2.2 implies that, for $r \in\left(0, r_{0}\right)$,

$$
\left|\left\langle z h^{\prime}(z), \frac{h(z)}{|h(z)|}\right\rangle\right|<|h(z)|
$$

i.e.,

$$
\left|\left\langle z f^{\prime}(z)-f(z), \frac{f(z)}{|f(z)|}\right\rangle\right|=\left|\left\langle z f^{\prime}(z), \frac{f(z)}{|f(z)|}\right\rangle-\right| f(z)||<|f(z)| .
$$

Especially, we have

$$
0<\operatorname{Re}\left\langle z f^{\prime}(z), \frac{f(z)}{|f(z)|}\right\rangle<2|f(z)|, \quad r \in\left(0, r_{0}\right) .
$$

Direct calculations show that

$$
r \frac{\partial}{\partial r}|f(z)|=\operatorname{Re}\left\langle z f^{\prime}(z), \frac{f(z)}{|f(z)|}\right\rangle, \quad f(z) \neq 0,
$$

and then we have

$$
0<r \frac{\partial}{\partial r}|f(z)|<2|f(z)|, \quad r \in\left(0, r_{0}\right)
$$

That is to say

$$
0<\frac{\partial}{\partial r} \log |f(z)|<\frac{2}{r}, \quad r \in\left(0, r_{0}\right) .
$$

Integrating the above inequality from $r_{1}$ to $r_{2}$ with $0<r_{1}<r_{2}<r_{0}$ gives that

$$
0<\log \frac{\left|f\left(r_{2} e^{i \theta}\right)\right|}{\left|f\left(r_{1} e^{i \theta}\right)\right|}<2 \log \frac{r_{2}}{r_{1}} .
$$

Hence, $\left|f\left(r e^{i \theta}\right)\right|$ and $r^{2} /\left|f\left(r e^{i \theta}\right)\right|$ are strictly increasing for $r \in\left(0, r_{0}\right)$. To see the sharpness of $r_{0}$, take the analytic function $f: \mathbf{D} \rightarrow \mathbf{C}^{n}$ given by

$$
f(z)=\left(z \frac{z+\alpha}{1+z \alpha}, 0, \ldots, 0\right), \quad z \in \mathbf{D} .
$$

The proof is complete.

Acknowledgements. The author is greatly indebted to three anonymous referees for their valuable suggestions which improve the presentation of the paper.

\section{References}

[1] Bisı, C.: A Landau's theorem in several complex variables. - Ann. Mat. Pura Appl. 196:2, 2017, 737-742.

[2] Bisi, C., and C. Stoppato: Landau's theorem for slice regular functions on the quaternionic unit ball. - Int. J. Math. 28:3, 2017, 1750017.

[3] Chen, H., and P. M. Gauthier: Bloch constants in several variables. - Trans. Amer. Math. Soc. 353:4, 2001, 1371-1386.

[4] Chen, H., and P. M. Gauthier: The Landau theorem and Bloch theorem for planar harmonic and pluriharmonic mappings. - Proc. Amer. Math. Soc. 139:2, 2011, 583-595.

[5] Chen, H., P. M. Gauthier, and W. Hengartner: Bloch constants for planar harmonic mappings. - Proc. Amer. Math. Soc. 128:11, 2000, 3231-3240.

[6] Chen, S., S. Ponnusamy, and X. Wang: Landau's theorem and Marden constant for harmonic $\nu$-Bloch mappings. - Bull. Aust. Math. Soc. 84:1, 2011, 19-32. 
[7] Dorff, M., and M. NowaK: Landau's theorem for planar harmonic mappings. - Comput. Methods Funct. Theory 4:1, 2004, 151-158.

[8] Graham, I., and G. Kohr: Geometric function theory in one and higher dimensions. Monogr. Textbooks Pure Appl. Math. 255, New York, 2003.

[9] Grigoryan, A.: Landau and Bloch theorems for harmonic mappings. - Complex Var. Elliptic Equ. 51:1, 2006, 81-87.

[10] Hamada, H., and G. Kohr: Pluriharmonic mappings in $\mathbf{C}^{n}$ and complex Banach spaces. J. Math. Anal. Appl. 426:2, 2015, 635-658.

[11] Huang, X.: Estimates on Bloch constants for planar harmonic mappings. - J. Math. Anal. Appl. 337:2, 2008, 880-887.

[12] Kalaj, D., and M. Vuorinen: On harmonic functions and the Schwarz lemma. - Proc. Amer. Math. Soc. 140:1, 2012, 161-165.

[13] Landau, E.: Der Picard-Schottysche Satz und die Blochsche Konstanten. - Sitzungsber Press Akad. Wiss. Berlin Phys.-Math. Kl., 1926, 467-474.

[14] Liu, M.: Estimates on Bloch constants for planar harmonic mappings. - Sci. China Ser. A $52: 1,2009,87-93$.

[15] PAvlović, M.: A Schwarz lemma for the modulus of a vector-valued analytic function. - Proc. Amer. Math. Soc. 139:3, 2011, 969-973.

[16] Rajala, K.: Bloch's theorem for mappings of bounded and finite distortion. - Math. Ann. $339: 2,2007,445-460$.

[17] Roper, K. A., and T. J. Suffridge: Convexity properties of holomorphic mappings in $\mathbf{C}^{n}$. - Trans. Amer. Math. Soc. 351:5, 1999, 1803-1833.

[18] XU, Z.: Schwarz lemma for pluriharmonic functions. - Indag. Math. (N.S.) 27:4, 2016, 923-929.

[19] XU, Z.: A Schwarz-Pick lemma for the norms of holomorphic mappings in Banach spaces. Complex Var. and Elliptic Equ. 63:10, 2018, 1459-1467.

[20] Xu, Z., and G. Ren: Slice starlike functions over quaternions. - J. Geom. Anal. 28:4, 2018, $3775-3806$.

[21] XU, Z., and X. WANG: On two Bloch type theorems for quaternionic slice regular functions. - arXiv:1601.02338, 2016.

Received 7 March $2020 \bullet$ Accepted 3 September 2020 